\title{
Feasibility of Using Two versus Three Rapid Tests for HIV Diagnosis in India: Analysis of Public Health Program Data
}

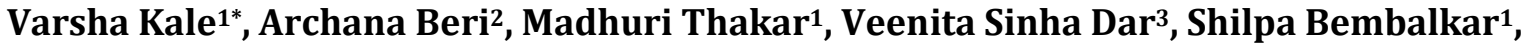 \\ Naresh Goel' ${ }^{3}$, Arun Risbud1', Ramesh Paranjape ${ }^{1}$ \\ ${ }^{1}$ National AIDS Research Institute, Pune, India \\ ${ }^{2}$ Centre for Disease Control and Prevention, New Delhi, India \\ ${ }^{3}$ National AIDS Control Organisation, Ministry of Health \& Family Welfare, New Delhi, India \\ Email: *kale_varsha123@yahoo.co.in
}

How to cite this paper: Kale, V., Beri, A., Thakar, M., Dar, V.S., Bembalkar, S., Goel, N., Risbud, A. and Paranjape, R. (2017) Feasibility of Using Two versus Three Rapid Tests for HIV Diagnosis in India: Analysis of Public Health Program Data. World Journal of AIDS, 7, 16-22. https://doi.org/10.4236/wja.2017.71002

Received: November 2, 2016

Accepted: January 23, 2017

Published: January 26, 2017

Copyright $\odot 2017$ by authors and Scientific Research Publishing Inc. This work is licensed under the Creative Commons Attribution International License (CC BY 4.0).

http://creativecommons.org/licenses/by/4.0/

(c) (i) Open Access

\begin{abstract}
Under India's National AIDS Control Program (NACP), WHO recommended strategy of using three rapid tests is adopted for diagnosis of HIV in an asymptomatic individual. Since the NACP has a stringent kit evaluation procedure and due to the availability of newer third generation Rapid Diagnostic Tests (RDTs) it may be possible to adapt two test strategy for HIV diagnosis instead of the recommended three test strategy. The authors reviewed programmatic data on HIV testing to explore whether use of two rapid tests for HIV diagnosis as against three tests could be a feasible approach without compromising the quality and readability of testing. Data on the HIV diagnosis performed on serum specimens collected for the period of one year (2011-12) from 82 Integrated Testing and Counselling Centers (ICTCs) or Prevention of Parent to Child Transmission Centers (PPTCTs) associated with State reference Laboratories (SRLs) was analysed. Out of 654258 results that were analysed 25168 (3.84\%) specimens were positives. It was observed that both two and three test algorithms provided similar results for majority ( $>99 \%)$ of the HIV positive specimens. Discordance was observed in labelling of specimen with inconclusive status $(\mathrm{N}=21)$, however the true status of these samples could not be obtained. The analysis indicated that the use of two test algorithm will have programmatic benefits in terms of reduced financial burden to the programme and ease of procurement, shipment and storage before and after distribution without comprising the quality of the testing. The prospective study would confirm this observation.
\end{abstract}

\section{Keywords}

HIV Testing, Two Test Strategy, Rapid Diagnostic Tests (RDT), National 


\section{Introduction/Background}

The mainstay of Human Immunodeficiency Virus (HIV) diagnosis is the detection of HIV specific antibodies in an individual. As per WHO 2015 HIV Testing guidelines, sequential testing of the sample for HIV specific antibodies by three Rapid Tests is recommended for confirmation of HIV diagnosis in settings of low HIV prevalence (less than 5\%) [1]. In India, under the National Acquired Immunodeficiency Disease Syndrome (AIDS) Control Program (NACP) the HIV diagnosis is carried out at number of the integrated counselling and testing centres (ICTC) and Prevention of Parent to Child Transmission centres (PPTCT). To ensure reliable testing, NACP has developed a large network of linkages between its ICTCs and 130 HIV testing reference laboratories for monitoring of quality through executing External Quality Assurance (EQA) Program and retesting mechanisms and training. Induction trainings are conducted for newly appointed laboratory staff at the testing centres. In addition, refresher trainings are conducted to keep staff updated on latest techniques and procedures.

All these testing centres use a three test strategy, where three RDTs are used sequentially for HIV diagnosis. Several RDTs that use recombinant and/or synthetic antigens are commercially available. The most commonly used RDTs are based on the principle of either immuno-concentration or dot blot immunoassay or immuno-chromatography. These tests are simple to perform and fast and easy to interpret. They do not require any special equipment to carry out the test. For this reason, RDTs have been used at the peripheral counselling and testing sites across different countries. This also has helped India to scale up HIV testing services in the national program to 22 million clients through 15606 testing centres in 2013-14 [2]. While most of the RDTs can differentiate between anti HIV-1 and HIV-2 antibodies, some of them are non-differentiating.

The three test strategy used for diagnosis of HIV infection in asymptomatic individuals at the ICTCs includes a screening test with high sensitivity (>99.5\%) and specificity above $98 \%$. [3]. The sample is considered negative if the screening test gives a nonreactive result. If the screening test is reactive, the sample is tested by two more tests. Three different kits with different antigen systems and/or different principles are used in the three test strategy. This strategy enables to identify "HIV positive", "HIV negative" or "HIV indeterminate" (Inconclusive status-reactive by two assays and nonreactive by one assay) status of an individual. The samples with "Indeterminate" results are sent to the reference laboratories to resolve the true status of the individual tested in context with HIV infection and also to identify quality related issues in testing if any. If the sample showing "indeterminate" result by RDT is found to be "Indeterminate" by western blot at the reference laboratory, patient is called back after two weeks for a retest to confirm HIV status. 
However, diagnosis using three test strategy is not free from challenges, importantly due to the challenges in the continuous supply of three different HIV kits to all the testing centres across the country. In a large country like India, where ICTCs are spread out over a large geographical area, transport of large number of kits is a tedious procedure. Storage of kits at ICTCs is also a problem due to shortage of space. The use of three tests pressurizes the procurement system for continuous availability of the three tests, also delays the diagnosis and reporting in absence of any one test. The two test algorithm could reduce this pressure and also the cost of the testing in the National programme. The turnaround time of reporting results will be reduced thus improving the efficiency of HIV testing centres. Also with advances in technology, the newer third generation diagnostic kits are performing better with higher sensitivity \& specificity.

Hence, it would be pertinent to assess if the two test strategy could be adopted in place of three test strategy for HIV diagnosis. The retrospective data on HIV testing was analysed in the present study for determining the ability of two test strategy to correctly identify HIV infected individuals compared to the three test strategy. For this, retrospective data on HIV testing of 654258 individuals at ICTCs/PPTCTs attached to 82 State Reference laboratories was collected for the period 2011-12. The study was intended to build evidence for NACP on whether two test strategy can be used in place of three test strategy without compromising the quality of testing.

\section{Methods}

Retrospective data on the HIV diagnosis performed on 654258 serum specimens collected over the period of one year (2011-2012) from 82 ICTCs or PPTCTs associated with State reference Laboratories was analysed. These sites are representative of all states of the country. The data of samples received from these laboratories was unlinked and anonymous with no identifier linked to any human subjects. The information on age, sex or risk group was unavailable for analysis.

In spite of large scale testing, quality standards were well maintained at these testing centres. The testing centres are under continuous quality control through EQA and retesting programmes and have successfully participated in EQA and retesting programme during the period of analysis. Additionally, utmost care is being taken in ensuring accuracy and reliability of kits used for HIV testing. Under NACP prior to procurement, the kits are being evaluated by a group of four laboratories forming a "Consortium for kit Quality" using a standardised SOP based on internationally accepted procedures. Kits successfully passed from Consortium for kit Quality only were procured for HIV diagnosis by the national programme.

The testing at the ICTCs/PPTCTs was performed using a standard strategy using three rapid tests (T1, T2 and T3) in a sequence as per the national guideline [3]. A number of rapid kits based on different principles have been used at the testing centres during the period of analysis. Test kits used at different centres are listed in Table 1. SD Bioline HIV1/2, Combaids RS Advantage, and 
Table 1. List of HIV kits used at centres during period of analysis.

\begin{tabular}{clll}
\hline Sr.No. & \multicolumn{1}{c}{ Name of kit } & \multicolumn{1}{c}{ Manufacturer } & \multicolumn{1}{c}{ Principle of test } \\
\hline 1 & Combaids - RS Advantage & Span diagnostics, Surat & Dot immunoassay \\
2 & Pareekshak HIV 1/2 Triline Card Test & Bhat Biotech, Bangalore & Immunochromatography \\
3 & Retrocheck HIV & Qualpro Diagnostics, Goa & Immunochromatography \\
4 & SD Bioline HIV 1/2 & Alere Medical Pvt. Ltd. Gurgaon & Immunochromatography \\
5 & Diagnos HIV BI-DOT & J. Mitra \& Co. Pvt. Ltd. New Delhi & Immunoconcentration \\
6 & INSTACHK HIV 1 + 2 & Transasia Bio-Medicals Ltd., Mumbai & Immunochromatography \\
7 & Pareekshak - Trispot & Bhat Biotech, Bangalore & Immunoconcentration \\
8 & Retroquic HIV & Qualpro Diagnostics, Goa & Immunoconcentration \\
9 & Retroscreen HIV & Qualpro Diagnostics, Goa & Immunochromatography \\
10 & HIV TRI DOT & J. Mitra \& Co. Pvt. Ltd. New Delhi & Immunoconcentration \\
11 & ACON & Acon Biotech (Hangzhou) Co., Ltd & Immunochromatography \\
12 & EIA COMB & J. Mitra \& Co. Pvt. Ltd. New Delhi & Enzymeimmunoassay \\
13 & Immunocomb II HIV 1 \& 2 Bispot & Orgenics, Israel & Enzymeimmunoassay
\end{tabular}

Pareekshak HIV1/2 Triline, are the most commonly used rapid kits. The three test strategy provided three outcomes or results viz. Positive, Negative and Indeterminate (Inconclusive). For two test strategy, the diagnosis based on the first two RDTs was considered and the result of the third test was disregarded for the purpose of analysis.

The case definitions for this analysis were taken as;

For Two test strategy (henceforth referred as 2TS):

HIV Positive: Defined as any specimen reactive with both, T1 and T2,

HIV Negative: Defined as any specimen nonreactive with T1 rapid test

HIV Indeterminate: Defined as a sample reactive by $\mathrm{T} 1$ and negative by $\mathrm{T} 2$

For Three test Strategy (henceforth referred as 3TS):

HIV Positive: Defined as any specimen reactive with all three T1, T2 and T3 rapid tests

HIV Negative: a) defined as any specimen nonreactive with T1 rapid test

b) defined as a sample reactive by the $\mathrm{T} 1$ and negative by both $\mathrm{T} 2$ and $\mathrm{T} 3$

HIV Indeterminate: defined as a sample reactive by $\mathrm{T} 1$ and nonreactive by either of $\mathrm{T} 2$ or $\mathrm{T} 3$.

Data from all ICTCs and PPTCTs were merged for the analysis. The number of HIV positives, HIV negatives and Indeterminates (inconclusive results) obtained by using two test and three test strategies were compared and the percentage of concordance was calculated. Results were summarized by cross tabulating HIV status by 2TS and 3TS. Also the cost of HIV testing by 2TS versus 3TS was compared.

\section{Results \& Discussion}

The analysis of the retrospective (data collected in 2011-12) HIV diagnosis data 
of 654258 specimens from ICTCs and PPTCTs is presented here. Out of a total 654258 entries, 25168 (3.84\%) results were positive, 629009 (96.1\%) negative and $81(0.01 \%)$ were Indeterminate (inconclusive) as per the 3TS. In comparison, 2TS labelled 25189 (3.85\%) results as positive, 628956 (96.1\%) as negative and $113(0.017 \%)$ as Indeterminate. (Inconclusive). Twenty one samples $(0.003 \%)$ were labelled as positive by $2 \mathrm{TS}$ which were reported as Indeterminate (inconclusive) by 3 TS. While 53 samples $(0.008 \%)$ were reported as Indeterminate (inconclusive) by 2TS, these samples were reported as negative by 3 TS (Table 2).

The analysis indicated that the 2TS correctly identified all HIV positive (25168) and all HIV negative (628956) samples as identified by 3TS. As per the set procedure in NACP, the 113 samples indeterminate by $2 \mathrm{TS}$ will be required to be tested by a confirmatory assay to assign correct status. Out of 113,60 were indeterminate by 3 TS also. Thus, additional 53 (0.008\%) samples were indeterminate by 2 TS which were negative by 3 TS. Also 21 samples $(0.003 \%)$ were reactive by first and second test and nonreactive by third test, hence labelled as positive by 2TS whereas indeterminate by 3TS. Since these patients did not turn up for follow up visit, there was no way to identify the correct status of these 21 samples.

This was one of the limitations of the retrospective analysis carried out in the study. Since the HIV diagnosis is associated with stigma and discrimination, the specificity of HIV testing is an important consideration in a public health setting. Hence, it is utmost important to address the false positivity if 2 TS is used. A prospective study should be considered to evaluate what percent of samples are likely to be falsely shown as positive by $2 \mathrm{TS}$.

The cost implication analysis was also carried out to understand the differences in the costs of 2TS and 3TS.

The analysis showed that the 2TS would require Rs. 3,60,866 (around US $\$ 5314$ ) less as compared to the cost of 3TS (3TS cost versus 2TS cost) (Table 3). This reduction in the cost of testing while using 2 TS is limited for 82 ICTCs/ PPTCTs considered for this analysis. Actually, in the year 2011-12, a total of $90,52,015$ individuals were tested for HIV in the national programme, out of which a total of 2,30,880 clients were found to be HIV positive. [4] If two test strategy had been used for testing these number of HIV positive clients, the reduction in the cost of testing would have been around Rs. 43,00,000 (US\$ 63328) which is quite significant in any resource limited setting including India and merits the importance of assessing two test strategy further.

Table 2. Comparison of 3TS with 2TS results.

\begin{tabular}{|c|c|c|c|c|c|}
\hline & & \multicolumn{4}{|c|}{$3 \mathrm{TS}$ results } \\
\hline & & Positive $(+++)$ & Negative (-) & Indeterminate (Inconclusive) & Total \\
\hline \multirow{4}{*}{$2 \mathrm{TS}$ results } & Positive $(++)$ & $25168(3.84 \%)$ & 0 & $21(++-)(0.003 \%)$ & $25189(3.85 \%)$ \\
\hline & Negative (-) & 0 & $628956(96.1 \%)$ & 0 & $628956(96.1 \%)$ \\
\hline & Indeterminate $(+-)$ & 0 & $53(0.008 \%)$ & $60(+-+)(0.009 \%)$ & $113(0.017 \%)$ \\
\hline & Total & $25168(3.84 \%)$ & 629009 (96.1\%) & $81(0.01 \%)$ & $654258(100 \%)$ \\
\hline
\end{tabular}


Table 3. Costs of the procurement of kits required for 2TS and 3TS.

\begin{tabular}{lccccc}
\hline \multicolumn{1}{c}{ No. of samples } & $\begin{array}{c}1^{\text {st }} \text { test } \\
\text { (Rs. 7.15 per test) }\end{array}$ & $\begin{array}{c}2^{\text {nd }} \text { test } \\
(\text { Rs. 14.74 per test })\end{array}$ & $\begin{array}{c}3^{\text {rd }} \text { test } \\
\text { (Rs. 18.55 per test) }\end{array}$ & $\begin{array}{c}\text { Western blot to assign correct status } \\
\text { (Rs. 2000 per test) }\end{array}$ & $\begin{array}{c}\text { Total cost } \\
(\text { Rs. })\end{array}$ \\
\hline $\begin{array}{l}\text { 2 TS - 25168 positives } \\
\text { plus 113 Indeterminates }\end{array}$ & 179951 & 370976 & Nil & 226000 & 776927 \\
$\begin{array}{l}\text { 3 TS - 25168 positives } \\
\text { plus 60 Indeterminates }\end{array}$ & 179951 & 370976 & 466866 & 120000 & 1137793 \\
\hline
\end{tabular}

The study showed that 2TS could give comparable results to 3TS without compromising quality and is less expensive. Swaminathan et al have also shown on a limited no of samples that two step rapid HIV testing algorithms could provide good cumulative sensitivity $(96.6 \%-98.6 \%)$ and specificity $(100 \%)$, and high positive and negative predictive values. [5]. However it should be kept in mind that the high PPV could be due to the nature of the HIV status of the tuberculosis patients analysed in this study. Since it was observed that the two test strategy might give false positive results, though in a small $(0.003 \%)$ percent of individuals, it would be worthwhile to further explore the utility of two test strategy in a planned prospective study.

Being programme data, there are limitations to the availability of data. However the strength lies in the large sample size used for analysis and reliability of the data due to the robust system under which the testing is being carried out.

\section{Conclusion}

2TS appears to be as good as 3TS for diagnosing HIV infection in programme setting. False positive results may be seen in a very small percentage of sample. This issue could not be definitively resolved due to non-availability of samples/data for further confirmation. The analysis indicates that it may be possible to replace 3TS by 2TS though additional evidence from a planned prospective study is needed before it is implemented in NACP.

\section{Acknowledgements}

The authors are thankful to all NRLS, SRLs and ICTCs/PPTCTs for providing HIV testing data.

\section{References}

[1] World Health Organisation Consolidated Guidelines on HIV Testing Services WHO Testing Guidelines July 2015. www.who.int/hiv/pub/guidelines/hiv-testing-services/en/

[2] Annual Report 2013-14, NACO, Department of AIDS Control, Ministry of Family Welfare, 50 . http://naco.gov.in/sites/default/files/NACO_English\%202013-14.pdf

[3] National Guidelines on HIV Testing, NACO. www.naco.gov.in/sites/.../National_Guidelines_for_HIV_Testing

[4] Annual Report 2011-12, NACO, Department of AIDS Control, Ministry of family welfare, 47. http://naco.gov.in/sites/default/files/NACO_AR_Eng\%202011-12.pdf 
[5] Swaminathan, S., Antony, L., Venkatesan, P., Hanna, L.E., Angayarkanni, B., et al. (2008) Sensitivity and Specificity of Combination Testing Algorithms for HIV in a Tuberculosis Clinic. Indian Journal of Medical Research, 128, 772-774.

Submit or recommend next manuscript to SCIRP and we will provide best service for you:

Accepting pre-submission inquiries through Email, Facebook, LinkedIn, Twitter, etc. A wide selection of journals (inclusive of 9 subjects, more than 200 journals)

Providing 24-hour high-quality service

User-friendly online submission system

Fair and swift peer-review system

Efficient typesetting and proofreading procedure

Display of the result of downloads and visits, as well as the number of cited articles

Maximum dissemination of your research work

Submit your manuscript at: http://papersubmission.scirp.org/

Or contactwja@scirp.org 